\title{
PROJECTIVE METRICS IN DYNAMIC PROGRAMMING
}

\author{
BY RICHARD BELLMAN AND THOMAS A. BROWN
}

Communicated by E. Hewitt, April 5, 1965

It has been shown by Birkhoff [2], [3] that Hilbert's projective metric [4] may be applied to a variety of problems involving linear mappings of a function space into itself. In this note we shall point out that essentially the same metric may be applied to some nonlinear mappings which frequently arise in dynamic programming [1].

Let $X$ be some set, and let $P$ denote the set of all nonnegative realvalued functions which have domain $X$ and are not identically zero. We define an extended real-valued function $\theta$ on $P \times P$ as follows:

$$
\theta(f, g)=\log \left[\left(\sup _{x \in \mathbb{X}} \frac{f(x)}{g(x)}\right) \cdot\left(\sup _{x \in \mathbf{X}} \frac{g(x)}{f(x)}\right)\right] .
$$

In computing the ratios, we take $0 \mid 0$ to be 1 , and $a \mid 0$ to be $\infty$ if $a \neq 0$. It is easy to show that $\theta$ is an extended pseudo-metric on $P$. $\theta(f, g)=0$ implies that $f=\lambda g$ for some constant $\lambda>0$. We say that a subset $P^{*}$ of $P$ is "metric" if $\theta$ is an extended metric on $P^{*}$. That is, if for any $f, g \in P^{*}, \theta(f, g)=0$ if and only if $f=g$.

Let $L$ be a map of $P$ into $P$. If

$$
\sup _{x \in X} \frac{L f(x)}{L g(x)}<\sup _{x \in X} \frac{f(x)}{g(x)} \text { for all } f, g \in P
$$

such that $0<\theta(f, g)<\infty$ then we say $L$ is "ratio reducing on $P$." Note that if $L$ is ratio reducing on $P$ it follows at once that $\theta(L f, L g)$ $<\theta(f, g)$ for all $f, g \in P$ such that $0<\theta(f, g)<\infty$.

Thus $L$ is a contraction mapping with respect to the pseudo-metric $\theta$. Similar definitions apply on any subset of $P$. Many linear transformations have been shown [2], [3] to be ratio reducing (or at least ratio nonincreasing). A family $\left\{L_{\lambda}\right\}$ ( $\lambda$ ranging over some set of parameters $\Lambda$ ) is said to be "uniformly ratio reducing" if, given $f, g$,

$$
\sup _{x \in X} \frac{L_{\lambda}(f(x))}{L_{\lambda}(g(x))} \leqq \sup _{x \in X} \frac{f(x)}{g(x)}-\delta_{f, 0} \text { for all } \lambda \in \Lambda,
$$

where $\delta_{f, o}>0$ may depend on $f$ and $g$ but does not depend on $\lambda$. Note that if $\Lambda$ is a finite set then the family $\left\{L_{\lambda}\right\}$ is uniformly ratio reducing if each of its members is ratio reducing.

THEOREM. If the family $\left\{L_{\lambda}: \lambda \in \Lambda\right\}$ is uniformly ratio reducing, 
then the transformation $L^{1}$ defined by

$$
L^{1}(f(x))=\sup _{\lambda \in \Lambda} L_{\lambda}(f(x))
$$

is ratio reducing. If in addition $L_{\lambda}(g(x))>\delta_{0}>0$ for each $g \in P$ and all $\lambda \in \Lambda$, then the transformation $L^{2}$ defined by

$$
L^{2}(f(x))=\inf _{\lambda \in \Delta} L_{\lambda}(f(x))
$$

is also ratio reducing.

The proof of the theorem is by straightforward computation. To illustrate the application of this theorem to dynamic programming, let us consider a class of problems referred to as "equations of type III" [1, pp. 125-129]. Suppose we are confronted with a system which may be in any one of $N+1$ states (call the states $s_{0}, s_{1}, \cdots, s_{N}$ ), and we are trying to drive the system in to state $s_{0}$. At each stage, we begin by knowing a probability distribution $p=\left(p_{0}, p_{1}, \cdots, p_{N}\right)$, where $p_{i}=$ probability that the system is in state $s_{i}$. We may either observe the system (at a cost $b>0$ ), or we may perform an operation $T_{i}$ on it which will alter the probability distribution in some way at a cost $a_{i}>0(i=1,2, \cdots, n)$. Then if $f(p)$ represents the expected cost of driving the system into state $s_{0}$ given that it is initially "known" to be in state $s_{i}$ with probability $p_{i}$, we see that $f$ must obey the functional equation

$$
f(p)=\inf \left\{\sum_{i=1}^{N} p_{i} f\left(\hat{s}_{i}\right)+b, f\left(T_{i} p\right)+a_{i}\right\}
$$

where $\hat{s}_{i}$ denotes the probability distribution which assigns probability 1 to state $s_{i}$.

THEOREM. There is at most one bounded positive solution to the equation $(*)$.

Proof. Let $X$ be the set of all possible distributions over the $N+1$ possible states with the exception of $(1,0, \cdots, 0)$. This point $\left(\hat{s}_{0}\right)$ is in the closure of $X$. Since the final operation on the system must be an observation, we see that $f(p) \geqq b$. If $f$ is bounded, it immediately follows that $\lim _{p \rightarrow s_{0}} f(p)=b$. Let us restrict our attention to the metric subset $P^{*}$ of $P$ consisting of bounded $f$ such that $\lim _{p \rightarrow \hat{s}_{0}} f(p)=b$.

$$
\begin{aligned}
& L_{0}(f(p))=\sum_{i=1}^{n} p_{i} f\left(\hat{s}_{i}\right)+b, \\
& L_{i}(f(p))=f\left(T_{i} p\right)+a_{i}, \quad i=1,2, \cdots, n,
\end{aligned}
$$


are all ratio-reducing on $P^{*}$. Thus by our Theorem above

$$
L(f(p))=\inf _{i=0,1, \ldots, n} L_{i}(f(p))
$$

is ratio-reducing on $P^{*}$. Hence, if $f$ and $g$ are distinct elements of $P^{*}$, then $\theta(L f, L g)<\theta(f, g)$, which proves there can be at most one bounded solution to $f=L f$.

A similar method may be applied when the system may be in any one of a continuum of states. Note that in addition to proving the uniqueness of the solution (if any) to $\left(^{*}\right)$, the above argument shows that if $g \in P^{*}$, and $\left\{L^{n} g\right\}$ contains a uniformly convergent subsequence, then $\left\{L^{n} g\right\}$ converges uniformly to the solution of $\left(^{*}\right)$.

\section{BIBLIOGRAPHY}

1. R. Bellman, Dynamic programming, Princeton Univ. Press, Princeton, N. J., 1957.

2. G. Birkhoff, Extensions of Jentzsch's theorem, Trans. Amer. Math. Soc. 85 (1957), 219-227.

3. - Uniformly semi-primitive multiplicative processes, Trans. Amer. Math. Soc. 104 (1962), 37-51.

4. D. Hilbert, Neue Begründung der Bolya-Lobatschefskyschen Geometrie, Math. Ann. 57 (1903), 137-150.

Rand Corporation, Santa Monica, California 